\title{
Group Analysis and New Explicit Solutions of Simplified Modified Kawahara Equation with Variable Coefficients
}

\author{
Gang-Wei Wang and Tian-Zhou Xu \\ School of Mathematics, Beijing Institute of Technology, Beijing 100081, PR, China \\ Correspondence should be addressed to Gang-Wei Wang; pukail121@163.com
}

Received 24 May 2013; Accepted 18 July 2013

Academic Editor: Teoman Özer

Copyright (C) 2013 G.-W. Wang and T.-Z. Xu. This is an open access article distributed under the Creative Commons Attribution License, which permits unrestricted use, distribution, and reproduction in any medium, provided the original work is properly cited.

The simplified modified Kawahara equation with variable coefficients is studied by using Lie symmetry method. Then we obtain the corresponding Lie algebra, optimal system, and the similarity reductions. At last, we also give some new explicit solutions for some special forms of the equations.

\section{Introduction}

Lie's classical theory of symmetries of differential equations is an inspiring source for various generalizations aiming to find the ways for obtaining explicit solutions. Lie's theory provides a standard method [1-5] for finding the Lie point symmetry group of a nonlinear system. And above all, Lie's method of infinitesimal transformation groups which essentially reduces the number of independent variables in partial differential equation (PDE) and reduces the order of ordinary differential equation (ODE) has been widely used in equations of mathematical physics. Lie method is an effective and the simplest method among group theoretic techniques and a large number of equations [6-11] are solved with the aid of this method.

In this paper, by using the Lie symmetry group method, we will consider the following simplified modified Kawahara equation:

$$
u_{t}+u^{2} u_{x}+\alpha(t) u+\beta(t) u_{x x x x x}=0 .
$$

Here in (1) the first term represents the evolution term while the second term represents the nonlinear term. The third term represents the linear damping $[6,7,12]$ while the fourth term is the dispersion term. The time dependent coefficients of damping and dispersion are, respectively, $\alpha(t)$ and $\beta(t)$ which are arbitrary smooth functions of the variable $t$. If $\alpha(t)=0$ and $\beta(t)=$ constant, (1) becomes the standard simplified modified Kawahara equation (see $[13,14]$ and references therein).
These KdV types of equations have been derived to model many physical phenomena, such as gravity-capillary waves on a shallow layer and magneto-sound propagation in plasmas, (see [14] and references therein). Many studies have been conducted with these types of equations [6-8, 12-18]. In [14] similarity solutions for some classes of (1) were considered. Abundant solitons solutions are obtained by using the tanh method in [17]. The paper [18] is mainly concerned with the local well-posedness of the initial-value problems for the Kawahara and the modified Kawahara equations in Sobolev spaces.

Our aim in the present work is to perform the variable coefficients version of the simplified modified Kawahara equation with the help of Lie's method. Then we get symmetry reductions and group-invariant solutions.

\section{Lie Group Classification}

2.1. Lie Symmetry Analysis of (1). In this section, we will perform Lie group method for (1).

If (1) is invariant under a one-parameter Lie group of point transformations

$$
\begin{aligned}
& t^{*}=t+\epsilon \tau(x, t, u)+O\left(\epsilon^{2}\right), \\
& x^{*}=x+\epsilon \xi(x, t, u)+O\left(\epsilon^{2}\right), \\
& u^{*}=u+\epsilon \eta(x, t, u)+O\left(\epsilon^{2}\right),
\end{aligned}
$$


with infinitesimal generator

$$
V=\tau(x, t, u) \frac{\partial}{\partial t}+\xi(x, t, u) \frac{\partial}{\partial x}+\eta(x, t, u) \frac{\partial}{\partial u},
$$

then the invariant condition reads as

$$
\begin{gathered}
\eta^{t}+u^{2} \eta^{x}+2 \eta u u_{x}+\alpha^{\prime}(t) \tau u+\beta^{\prime}(t) \tau u_{x x x x x} \\
+\alpha(t) \eta+\beta(t) \eta^{x x x x x}=0
\end{gathered}
$$

where

$$
\begin{gathered}
\eta^{t}=D_{t}(\eta)-u_{x} D_{t}(\xi)-u_{t} D_{t}(\tau), \\
\eta^{x}=D_{x}(\eta)-u_{x} D_{x}(\xi)-u_{t} D_{x}(\tau), \\
\eta^{x x}=D_{x}\left(\eta^{x}\right)-u_{x t} D_{x}(\tau)-u_{x x} D_{x}(\xi), \\
\eta^{x x x}=D_{x}\left(\eta^{x x}\right)-u_{x x t} D_{x}(\tau)-u_{x x x} D_{x}(\xi), \\
\eta^{x x x x}=D_{x}\left(\eta^{x x x}\right)-u_{x x x t} D_{x}(\tau)-u_{x x x x} D_{x}(\xi), \\
\eta^{x x x x x}=D_{x}\left(\eta^{x x x x}\right)-u_{x x x x t} D_{x}(\tau)-u_{x x x x x} D_{x}(\xi) .
\end{gathered}
$$

Here, $D_{i}$ denotes the total derivative operator and is defined by

$$
D_{i}=\frac{\partial}{\partial x^{i}}+u_{i} \frac{\partial}{\partial u}+u_{i j} \frac{\partial}{\partial u_{j}}+\cdots, \quad i=1,2,
$$

and $\left(x^{1}, x^{2}\right)=(t, x)$.

Solving (4) with the help of (5) we obtain

$$
\begin{gathered}
\tau=\tau(t), \quad \xi_{u}=0, \quad \eta_{u u}=0, \\
\beta(t) \tau_{t}+\beta^{\prime}(t) \tau-5 \beta(t) \xi_{x}=0, \\
\eta_{x u}-2 \xi_{x x}=0, \\
\tau_{t} u^{2}-\xi_{x} u^{2}+2 \eta u-\xi_{t}+\beta(t)\left(5 \eta_{x x x x u}-\xi_{x x x x x}\right)=0, \\
\eta_{t}+\alpha(t) \eta+\alpha(t) u \tau_{t}-\alpha(t) u \eta_{u} \\
+u \alpha^{\prime}(t) \tau+u^{2} \eta_{x}+\beta(t) \eta_{x x x x x}=0 .
\end{gathered}
$$

The structure of the determining equations (10) and (11) may give the selection of the following three forms for the coefficient $\alpha(t)$.

Case $1(\alpha(t)=0)$. In this case, solving (7)-(11) we get

$$
\begin{gathered}
\xi=2 c_{1} x+c_{2}, \quad \tau=2 c_{3} t+c_{4}, \\
\eta=\left(c_{1}-c_{3}\right) u
\end{gathered}
$$

and (8) becomes

$$
\beta^{\prime}(t)\left(2 c_{3} t+c_{4}\right)+\beta(t)\left(2 c_{3}-10 c_{1}\right)=0,
$$

where $c_{1}, \ldots, c_{4}$ are constants. The analysis of (13) leads to the following three possibilities for $\beta(t)$.
(1.1) $\beta(t)$ is arbitrary.

For this case, we obtain the vector field

$$
V_{1}=\frac{\partial}{\partial x}
$$

(1.2) $\beta(t)=b_{0}(\neq 0)$ is a constant.

Equation (1) admits a three-dimensional Lie algebra spanned by

$$
\begin{gathered}
V_{1}=\frac{\partial}{\partial x}, \quad V_{2}=\frac{\partial}{\partial t} \\
V_{3}=x \frac{\partial}{\partial x}+5 t \frac{\partial}{\partial t}-2 u \frac{\partial}{\partial u} .
\end{gathered}
$$

(1.3) $\beta(t)=b_{0} \mathrm{e}^{m t}, b_{0}, m(\neq 0)$ are constant.

We obtain the corresponding two Lie point symmetry generators

$$
V_{1}=\frac{\partial}{\partial x}, \quad V_{2}=\frac{\partial}{\partial t}+\frac{m}{5} x \frac{\partial}{\partial x}+\frac{m}{10} u \frac{\partial}{\partial u}
$$

Case $2(\alpha(t)$ is a nonzero constant as $a)$. Similarly, in this case, solving (7)-(11) for the infinitesimals, we obtain

$$
\begin{gathered}
\xi=2 c_{1} x+c_{3}, \quad \tau=-\frac{c_{2}}{a} \mathrm{e}^{2 a t}+c_{4}, \\
\eta=\left(c_{1}+c_{2} \mathrm{e}^{2 a t}\right) u,
\end{gathered}
$$

and (8) becomes

$$
\beta^{\prime}(t)\left(-\frac{c_{2}}{a} \mathrm{e}^{2 a t}+c_{4}\right)+\beta(t)\left(-2 c_{2} \mathrm{e}^{2 a t}-10 c_{1}\right)=0,
$$

where $c_{1}, \ldots, c_{4}$ are constants. The analysis of (18) gives rise to the following four possibilities for $\beta(t)$.

(2.1) $\beta(t)$ is arbitrary.

For this case, we obtain the vector field

$$
V_{1}=\frac{\partial}{\partial x}
$$

(2.2) $\beta(t)=b_{0}(\neq 0)$ is a constant.

We have the corresponding two-dimensional Lie algebra

$$
V_{1}=\frac{\partial}{\partial x}, \quad V_{2}=\frac{\partial}{\partial t}
$$


(2.3) $\beta(t)=b_{0} \mathrm{e}^{-2 a t}, b_{0}$ is a constant.

Equation (1) admits a three-dimensional Lie algebra spanned by

$$
\begin{gathered}
V_{1}=\frac{\partial}{\partial x}, \quad V_{2}=\mathrm{e}^{2 a t} \frac{\partial}{\partial t}-a \mathrm{e}^{2 a t} u \frac{\partial}{\partial u}, \\
V_{3}=5 \frac{\partial}{\partial t}-2 a x \frac{\partial}{\partial x}-a u \frac{\partial}{\partial u} .
\end{gathered}
$$

(2.4) $\beta(t)=b_{0} \mathrm{e}^{m t}\left(b_{0} \neq 0\right)$ is a constant.

Substituting $\beta(t)$ into (18), one can get

$$
c_{2} \mathrm{e}^{2 a t}\left(\frac{m}{a}+2\right)=10 c_{1}-c_{4} m
$$

where $m \neq-2 a$. We obtain the corresponding twodimensional Lie algebra

$$
\begin{gathered}
V_{1}=\frac{\partial}{\partial x} \\
V_{2}=\frac{\partial}{\partial t}+\frac{m}{5} x \frac{\partial}{\partial x}+\frac{m}{10} u \frac{\partial}{\partial u} .
\end{gathered}
$$

Case $3(\alpha(t)=1 / t)$. In this case, solving (7)-(11), we get

$$
\begin{gathered}
\xi=c_{3} x+c_{4}, \quad \tau=-c_{1} t^{2}-2 c_{2} t+c_{3} t, \\
\eta=\left(c_{1} t+c_{2}\right) u,
\end{gathered}
$$

and (8) becomes

$$
\beta^{\prime}(t)\left(-c_{1} t^{2}-2 c_{2} t+c_{3} t\right)+\beta(t)\left(-4 c_{3}-2 c_{2}-2 c_{1} t\right)=0,
$$

where $c_{1}, \ldots, c_{4}$ are constants. Similarly, the analysis of (25) gives rise to the following three possibilities for $\beta(t)$.

(3.1) $\beta(t)$ is arbitrary.

For this case, we obtain the vector field

$$
V_{1}=\frac{\partial}{\partial x}
$$

(3.2) $\beta(t)=b_{0}(\neq 0)$ is a constant. bra

We obtain the corresponding two-dimensional Lie alge-

$$
V_{1}=\frac{\partial}{\partial x}, \quad V_{2}=x \frac{\partial}{\partial x}+5 t \frac{\partial}{\partial t}-2 u \frac{\partial}{\partial u}
$$

(3.3) $\beta(t)=b_{0} / t^{2}, b_{0}(\neq 0)$ is a constant.

The Lie algebra is extended by the symmetry generators

$$
\begin{gathered}
V_{1}=\frac{\partial}{\partial x}, \quad V_{2}=t^{2} \frac{\partial}{\partial t}-t u \frac{\partial}{\partial u}, \\
V_{3}=x \frac{\partial}{\partial x}-5 t \frac{\partial}{\partial t}+3 u \frac{\partial}{\partial u} .
\end{gathered}
$$

TABLE 1: Commutator table of the Lie algebra of (1.2).

\begin{tabular}{lccc}
\hline$\left[V_{i}, V_{j}\right]$ & $V_{1}$ & $V_{2}$ & $V_{3}$ \\
\hline$V_{1}$ & 0 & 0 & $V_{1}$ \\
$V_{2}$ & 0 & 0 & $5 V_{2}$ \\
$V_{3}$ & $-V_{1}$ & $-5 V_{2}$ & 0 \\
\hline
\end{tabular}

TABLE 2: Adjoint table of the Lie algebra of (1.2).

\begin{tabular}{cccc}
\hline Ad & $V_{1}$ & $V_{2}$ & $V_{3}$ \\
\hline$V_{1}$ & $V_{1}$ & $V_{2}$ & $V_{3}-\epsilon V_{1}$ \\
$V_{2}$ & $V_{1}$ & $V_{2}$ & $V_{3}-5 \epsilon V_{2}$ \\
$V_{3}$ & $e^{\epsilon} V_{1}$ & $e^{5 \epsilon} V_{2}$ & $V_{3}$ \\
\hline
\end{tabular}

TABle 3: Commutator table of the Lie algebra of (2.3).

\begin{tabular}{lccc}
\hline$\left[V_{i}, V_{j}\right]$ & $V_{1}$ & $V_{2}$ & $V_{3}$ \\
\hline$V_{1}$ & 0 & 0 & $-2 a V_{1}$ \\
$V_{2}$ & 0 & 0 & $-10 a V_{2}$ \\
$V_{3}$ & $2 a V_{1}$ & $10 a V_{2}$ & 0 \\
\hline
\end{tabular}

2.2. Optimal System of One-Dimensional Lie Algebras. First of all, we briefly review the main definitions [1] which will be used in the following sections.

Definition 1 (see [1]). Let $G$ be a Lie group. An optimal system of $s$-parameter subgroups is a list of conjugacy inequivalent $s$-parameter subgroups with the property that any other subgroup is conjugate to precisely one subgroup in the list. Similarly, a list of $s$-parameter subalgebras forms an optimal system if every $s$-parameter subalgebra of $g$ is equivalent to a unique member of the list under some element of the adjoint representation: $\hbar=A d g(h), g \in G$.

To obtain the optimal system, we apply the formula [1]

$$
\operatorname{Ad}\left(\exp \left(\epsilon V_{i}\right)\right) V_{j}=V_{j}-\epsilon\left[V_{i}, V_{j}\right]+\frac{1}{2} \epsilon^{2}\left[V_{i},\left[V_{i}, V_{j}\right]\right]-\cdots,
$$

where $\epsilon$ is a real constant. Here $\left[V_{i}, V_{j}\right]$ is the commutator for the Lie algebra given by

$$
\left[V_{i}, V_{j}\right]=V_{i} V_{j}-V_{j} V_{i}
$$

The commutator table of the Lie point symmetries of (1.2) and the adjoint representations of the symmetry group of (1.2) on its Lie algebra are given in Tables 1 and 2, respectively. In the same way, the commutator table and the adjoint representations of (2.3), (2.4), and (3.3) are given in Tables 3, $4,5,6,7$, and 8 , respectively. We give in Table 9 optimal system of subalgebras for (1.2), (2.3), (2.4), and (3.3), respectively.

Remark 2. For simplicity, we will refer to the equation corresponding to the case (1.3) as (1.3), and so on.

Remark 3. For brevity we only consider the optimal system for the equation concerned in case (1.2) in detail and the rest will be listed in Table 9 as they can be derived in a similar manner. 
TABLE 4: Adjoint table of the Lie algebra of (2.3).

\begin{tabular}{lccc}
\hline Ad & $V_{1}$ & $V_{2}$ & $V_{3}$ \\
\hline$V_{1}$ & $V_{1}$ & $V_{2}$ & $V_{3}+2 a \epsilon V_{1}$ \\
$V_{2}$ & $V_{1}$ & $V_{2}$ & $V_{3}+10 a \epsilon V_{2}$ \\
$V_{3}$ & $e^{-2 a \epsilon V_{1}}$ & $e^{-10 a \epsilon} V_{2}$ & $V_{3}$ \\
\hline
\end{tabular}

TABLE 5: Commutator table of the Lie algebra of (2.4).

\begin{tabular}{lcc}
\hline$\left[V_{i}, V_{j}\right]$ & $V_{1}$ & $V_{2}$ \\
\hline$V_{1}$ & 0 & 0 \\
$V_{2}$ & 0 & 0 \\
\hline
\end{tabular}

TABLE 6: Adjoint table of the Lie algebra of (2.4).

\begin{tabular}{lll}
\hline Ad & $V_{1}$ & $V_{2}$ \\
\hline$V_{1}$ & $V_{1}$ & $V_{2}$ \\
$V_{2}$ & $V_{1}$ & $V_{2}$ \\
\hline
\end{tabular}

TABLE 7: Commutator table of the Lie algebra of (3.3).

\begin{tabular}{lccc}
\hline$\left[V_{i}, V_{j}\right]$ & $V_{1}$ & $V_{2}$ & $V_{3}$ \\
\hline$V_{1}$ & 0 & 0 & $V_{1}$ \\
$V_{2}$ & 0 & 0 & $5 V_{2}$ \\
$V_{3}$ & $-V_{1}$ & $-5 V_{2}$ & 0 \\
\hline
\end{tabular}

TABLE 8: Adjoint table of the Lie algebra of (3.3).

\begin{tabular}{lccc}
\hline Ad & $V_{1}$ & $V_{2}$ & $V_{3}$ \\
\hline$V_{1}$ & $V_{1}$ & $V_{2}$ & $V_{3}-\epsilon V_{1}$ \\
$V_{2}$ & $V_{1}$ & $V_{2}$ & $V_{3}-5 \epsilon V_{2}$ \\
$V_{3}$ & $e^{\epsilon} V_{1}$ & $e^{5 \epsilon} V_{2}$ & $V_{3}$ \\
\hline
\end{tabular}

\section{Symmetry Reductions and Exact Group-Invariant Solutions}

In this section, we will use Table 9 to obtain symmetry reductions and exact group-invariant solutions for (1.2), (2.3), (2.4), and (3.3).

\subsection{Symmetry Reductions and Exact Solutions to (1.2)}

3.1.1. $V_{1}$. For the generator $V_{1}$, the group-invariant solution is $u=f(\xi)$, where $\xi=t$ is the group-invariant, the substitution of this solution into (1.2) gives the trivial solution $u(x, t)=C$, and $C$ is a constant.

3.1.2. $V_{2}+\lambda V_{1}$. For the linear combination $V_{2}+\lambda V_{1}$, we have

$$
u=f(\xi)
$$

where $\xi=x-\lambda t$ is the group-invariant. Substituting (31) into (1.2), we reduce it to the following ODE:

$$
b_{0} f^{(5)}+f^{2} f^{\prime}-\lambda f^{\prime}=0
$$

3.1.3. $V_{3}$. For the generator $V_{3}$, we have

$$
u=t^{-(2 / 5)} f(\xi)
$$

where $\xi=x t^{-(1 / 5)}$ is the group-invariant. Substituting (33) into (1.2), we reduce it to the following ODE:

$$
5 b_{0} f^{(5)}+5 f^{2} f^{\prime}-2 f-\xi f^{\prime}=0
$$

where $f^{\prime}=d f / d \xi$.

\subsection{Symmetry Reductions and Exact Solutions to (2.3)}

3.2.1. $V_{1}$. For the generator $V_{1}$, we get that the groupinvariant solution of $(2.3)$ is $u(x, t)=c e^{-a t}$, where $c$ is an arbitrary constant.

3.2.2. $V_{3}$. For the generator $V_{3}$, we have

$$
u=e^{-a t / 5} f(\xi)
$$

where $\xi=x e^{2 a t / 5}$. Substituting (35) into (2.3), one can get

$$
5 b_{0} f^{(5)}+5 f^{2} f^{\prime}+4 a f+2 a \xi f^{\prime}=0
$$

where $f^{\prime}=d f / d \xi$.

3.2.3. $V_{2}+\mu V_{1}$. (i) For $\mu \neq 0$, we have

$$
u=f(\xi) \mathrm{e}^{-a t},
$$

where $\xi=x+\left(\mu \mathrm{e}^{-2 a t} / 2 a\right)$. Substituting (37) into (2.3) yields

$$
b_{0} f^{(5)}+f^{2} f^{\prime}-\mu f^{\prime}=0 .
$$

(ii) For $\mu=0$, we get

$$
b_{0} f^{(5)}+f^{2} f^{\prime}=0
$$

\subsection{Symmetry Reductions and Exact Solutions to (2.4)}

3.3.1. $V_{1}$. For the generator $V_{1}$, we get that the groupinvariant solution of (2.4) is

$$
u(x, t)=c e^{-a t}
$$

where $c$ is an arbitrary constant.

3.3.2. $V_{2}$. For this case, we have

$$
\xi=x \mathrm{e}^{-(m t / 5)}, \quad u=\mathrm{e}^{m t / 10} f(\xi)
$$

then substituting (41) into (2.4) gives rise to

$$
10 b_{0} f^{(5)}+10 f^{2} f^{\prime}+10 a f-2 m \xi f^{\prime}+m f=0 .
$$


TABLE 9: Optimal system of subalgebras of some special cases of (1.1).

\begin{tabular}{lcr}
\hline Equation number & Equations & Optimal system \\
\hline$(1.2)$ & $u_{t}+u^{2} u_{x}+b_{0} u_{x x x x x}=0$ & $V_{1}, V_{2}+\lambda V_{1}, V_{3}$ \\
$(2.3)$ & $u_{t}+u^{2} u_{x}+a u+\left(b_{0} e^{-2 a t}\right) u_{x x x x x}=0$ & $V_{1}, V_{2}+\mu V_{1}, V_{3}$ \\
$(2.4)$ & $u_{t}+u^{2} u_{x}+a u+\left(b_{0} e^{m t}\right) u_{x x x x x}=0$ & $V_{1}, V_{2}$ \\
$(3.3)$ & $u_{t}+u^{2} u_{x}+(1 / t) u+\left(b_{0} / t^{2}\right) u_{x x x x x}=0$ & $V_{1}, V_{2}+\mu V_{1}, V_{3}$ \\
\hline
\end{tabular}

Here $\mu=0, \mu= \pm 1$, and $\lambda$ is a nonzero constant.

\subsection{Symmetry Reductions and Exact Solutions to (3.3)}

3.4.1. $V_{1}$. For the generator $V_{1}$, we get that the groupinvariant solution of (3.3) is

$$
u(x, t)=\frac{c}{t}
$$

where $c$ is an arbitrary constant.

3.4.2. $V_{3}$. For the generator $V_{3}$, we have

$$
u=t^{-(3 / 5)} f(\xi)
$$

where $\xi=x t^{1 / 5}$. Substituting (44) into (3.3), one can get

$$
5 b_{0} f^{(5)}+5 f^{2} f^{\prime}+2 f+\xi f^{\prime}=0
$$

where $f^{\prime}=d f / d \xi$.

3.4.3. $V_{2}+\mu V_{1}$. (i) For $\mu \neq 0$, we have

$$
u=\frac{1}{t} f(\xi)
$$

where $\xi=x+\mu / t$. Substituting (46) into (3.3) yields

$$
b_{0} f^{(5)}+f^{2} f^{\prime}-\mu f^{\prime}=0 .
$$

In order to search for other explicit solutions, by using the Jacobi elliptic function expansion method [19]. By virtue of the technique of solution we introduce the ansatz

$$
f=a_{0}+a_{1} \operatorname{sn}(\xi)+a_{2} \operatorname{sn}^{2}(\xi) .
$$

Substituting (48) into (47), one can get

$$
\begin{gathered}
b=-\frac{1}{360} \frac{a_{2}^{2}}{m^{4}}, \quad m=m, \quad \mu=\frac{1}{15} \frac{a_{2}^{2}\left(-m^{2}+1+m^{4}\right)}{m^{4}}, \\
a_{0}=-\frac{1}{3} \frac{a_{2}\left(1+m^{2}\right)}{m^{2}}, \quad a_{1}=0, \quad a_{2}=a_{2} .
\end{gathered}
$$

Thus, we obtain Jacobi elliptic function solutions of (3.3) as follows:

$$
u(x, t)=-\frac{1}{3} \frac{a_{2}\left(1+m^{2}\right)}{m^{2}}+a_{2} \operatorname{sn}^{2}(\xi) .
$$

In particular, when $m \rightarrow 1$, we can obtain hyperbolic function solutions

$$
u(x, t)=-\frac{1}{3} \frac{a_{2}\left(1+m^{2}\right)}{m^{2}}+a_{2} \tanh ^{2}(\xi)
$$

When $m \rightarrow 0$, we can obtain trigonometric function solutions

$$
u(x, t)=-\frac{1}{3} \frac{a_{2}\left(1+m^{2}\right)}{m^{2}}+a_{2} \sin ^{2}(\xi),
$$

where $\xi=x+\mu / t$

(ii) For $\mu=0$, we get

$$
b_{0} f^{(5)}+f^{2} f^{\prime}=0 \text {. }
$$

Remark 4. It is not difficult to find out that the reduced ODEs may be classified into four classes:

$$
\begin{gathered}
f^{(5)}+A f^{2} f^{\prime}=0, \\
f^{(5)}+A f^{2} f^{\prime}+B f^{\prime}=0, \\
f^{(5)}+A f^{2} f^{\prime}+B f^{\prime}+C f=0, \\
f^{(5)}+A f^{2} f^{\prime}+B f^{\prime}+C \xi f^{\prime}=0 .
\end{gathered}
$$

\section{The Explicit Power Series Solutions}

In this section, we will consider the explicit analytic solutions of some special forms of reduced equations by using the power series method.

Now, we seek a solution of (45) in a power series of the following form:

$$
f(\xi)=\sum_{n=0}^{\infty} c_{n} \xi^{n}
$$


Substituting (55) into (45), we get

$$
\begin{aligned}
600 b c_{5} & +b \sum_{n=1}^{\infty}(n+1)(n+2)(n+3)(n+4)(n+5) c_{n+5} \xi^{n} \\
& +5 c_{0}^{2} c_{1}+5 \sum_{n=1}^{\infty} \sum_{k=0}^{n} \sum_{j=0}^{k}(n+1-k) c_{j} c_{k-j} c_{n+1-k} \xi^{n} \\
& +2 c_{0}+2 \sum_{n=1}^{\infty} c_{n} \xi^{n} \\
& +\sum_{n=1}^{\infty} n c_{n} \xi^{n}=0 .
\end{aligned}
$$

Now from (56), comparing coefficients, for $n=0$, one can get

$$
c_{5}=-\frac{2 c_{0}+5 c_{0}^{2} c_{1}}{600 b} \text {. }
$$

Generally, for $n \geq 1$, we obtain

$$
\begin{aligned}
c_{n+5}= & -\frac{1}{b(n+1)(n+2)(n+3)(n+4)(n+5)} \\
& \times\left((n+2) c_{n}+5 \sum_{k=0}^{n} \sum_{j=0}^{k}(n+k-1) c_{j} c_{k-j} c_{n+1-k}\right) .
\end{aligned}
$$

From (57) and (58), we can obtain all the coefficients $c_{n}(n \geq 5)$ of the power series (55). For arbitrary chosen constant numbers $c_{0}, c_{1}, c_{2}, c_{3}$, and $c_{4}$, the other terms also can be determined successively from (57) and (58) in a unique way. In addition, it is easy to prove the convergence of the power series (55) with the coefficients given by (57) and (58) $[20,21]$. The details are omitted here. In this connection, this power series solution is an explicit analytic solution.

So, the power series solution of (45) can be written as follows:

$$
\begin{aligned}
f(\xi)= & c_{0}+c_{1} \xi+c_{2} \xi^{2}+c_{3} \xi^{3} \\
& +c_{4} \xi^{4}+c_{5} \xi^{5}+\sum_{n=1}^{\infty} c_{n+5} \xi^{n+5} \\
= & c_{0}+c_{1} \xi+c_{2} \xi^{2}+c_{3} \xi^{3}+c_{4} \xi^{4} \\
& -\frac{2 c_{0}+5 c_{0}^{2} c_{1}}{600 b} \xi^{5} \\
& -\sum_{n=0}^{\infty} \frac{1}{b(n+1)(n+2)(n+3)(n+4)(n+5)}
\end{aligned}
$$

$$
\begin{aligned}
& \times\left((n+2) c_{n}\right. \\
& \left.\quad+5 \sum_{k=0}^{n} \sum_{j=0}^{k}(n+k-1) c_{j} c_{k-j} c_{n+1-k}\right) \xi^{n+5} .
\end{aligned}
$$

Thus, the exact power series solution of (3.3) is

$$
\begin{aligned}
& u(x, t)=\left[c_{0}+c_{1}\left(x t^{1 / 5}\right)+c_{2}\left(x t^{1 / 5}\right)^{2}\right. \\
& +c_{3}\left(x t^{1 / 5}\right)^{3}+c_{4}\left(x t^{1 / 5}\right)^{4} \\
& -\frac{2 c_{0}+5 c_{0}^{2} c_{1}}{600 b}\left(x t^{1 / 5}\right)^{5} \\
& \left.+\sum_{n=0}^{\infty} c_{n+5}\left(x t^{1 / 5}\right)^{n+5}\right]\left(t^{-(3 / 5)}\right) \\
& =\left[c_{0}+c_{1}\left(x t^{1 / 5}\right)+c_{2}\left(x t^{1 / 5}\right)^{2}\right. \\
& +c_{3}\left(x t^{1 / 5}\right)^{3}+c_{4}\left(x t^{1 / 5}\right)^{4} \\
& -\frac{2 c_{0}+5 c_{0}^{2} c_{1}}{600 b}\left(x t^{1 / 5}\right)^{5} \\
& -\sum_{n=0}^{\infty} \frac{1}{b(n+1)(n+2)(n+3)(n+4)(n+5)} \\
& \times\left((n+2) c_{n}\right. \\
& \left.+5 \sum_{k=0}^{n} \sum_{j=0}^{k}(n+k-1) c_{j} c_{k-j} c_{n+1-k}\right) \\
& \left.\times \xi^{n+5}\right]\left(t^{-(3 / 5)}\right)
\end{aligned}
$$

where $c_{i}(i=0,1,2,3,4)$ are arbitrary constants, and the other coefficients $c_{n}(n \geq 5)$ can be determined successively from (57) and (58).

Of course, in physical applications, it will be convenient to write the solution of (45) in the approximate form

$$
\begin{aligned}
u(x, t)= & {\left[c_{0}+c_{1}\left(x t^{1 / 5}\right)+c_{2}\left(x t^{1 / 5}\right)^{2}+c_{3}\left(x t^{1 / 5}\right)^{3}\right.} \\
& \left.\quad+c_{4}\left(x t^{1 / 5}\right)^{4}-\frac{2 c_{0}+5 c_{0}^{2} c_{1}}{600 b}\left(x t^{1 / 5}\right)^{5}+\cdots\right] \\
& \times\left(t^{-(3 / 5)}\right) .
\end{aligned}
$$


Remark 5. The exact solution of the rest of equations can be derived in a similar manner. We have details omitted here.

Remark 6. It is easy to see that the reduced equations (54) are all higher-order nonlinear ODEs or with nonconstant coefficients. If we obtain a one-parameter symmetry group of an ODE, then we could reduce the order of the equation by one. However, we find out that such reduced ODEs are more complicated than the original equation. In general, we cannot obtain the exact explicit solutions for higherorder nonlinear ODEs or with nonconstant coefficients by using the elementary functions and integrals. However, the power series can be used to solve them. In view of this, we can find that the power series method [14, 15, 20-23] is an effective tool of solving such ODEs. Moreover, from our model, we could find that these power series solutions are important for computations in numerical analysis and physical applications. And above all, these power series play an important role in the investigation of physical phenomena and other natural phenomena.

Remark 7. Indeed, for all the rest of the cases presented in Section 2, it is possible to get optimal systems and symmetry reductions, but for brevity we have omitted them here.

\section{Conclusions}

We have performed Lie symmetry analysis for the simplified modified Kawahara equation with variable coefficients. Then the similarity reductions and exact solutions are obtained based on the optimal system of the one-dimensional Lie algebras, for some special forms of the equations. Moreover, the power series solution of the reduced equation are given simultaneously. These are new solutions for the simplified modified Kawahara equation with variable coefficients. The symmetry analysis based on the Lie group method is a very powerful method and is worthy of being studyied further.

\section{Acknowledgments}

The project is supported by the National Natural Science Foundation of China (NNSFC) (Grant no. 11171022). The authors express their sincere thanks to the referees for their careful review of this paper and their useful suggestions.

\section{References}

[1] P. J. Olver, Application of Lie Group to Differential Equation, Springer, New York, NY, USA, 1986.

[2] L. V. Ovsiannikov, Group Analysis of Differential Equations, Academic Press, New York, NY, USA, 1982.

[3] S. Lie, "On integration of a class of linear partial differential equations by means of definite integrals," Archive for Mathematical Logic, vol. 6, no. 3, pp. 328-368, 1881.

[4] G. W. Bluman and S. Kumei, Symmetries and Differential Equations, vol. 81 of Applied Mathematical Sciences, Springer, New York, NY, USA, 1989.
[5] N. H. Ibragimov, Ed., CRC Handbook of Lie Group Analysis of Differential Equations, vol. 1-3, CRC Press, Boca Raton, Fla, USA, 1994.

[6] A. G. Johnpillai and C. M. Khalique, "Group analysis of KdV equation with time dependent coefficients," Applied Mathematics and Computation, vol. 216, no. 12, pp. 3761-3771, 2010.

[7] A. G. Johnpillai and C. M. Khalique, "Lie group classification and invariant solutions of $\mathrm{mKdV}$ equation with time-dependent coefficients," Communications in Nonlinear Science and Numerical Simulation, vol. 16, no. 3, pp. 1207-1215, 2011.

[8] G. W. Wang, X. Q. Liu, and Y. Y. Zhang, "Lie symmetry analysis to the time fractional generalized fifth-order KdV equation," Communications in Nonlinear Science and Numerical Simulation, vol. 18, pp. 2321-2326, 2013.

[9] G. W. Wang, X. Q. Liu, and Y. Y. Zhang, "Symmetry reduction, exact solutions and conservation laws of a new fifth-order nonlinear integrable equation," Communications in Nonlinear Science and Numerical Simulation, vol. 18, pp. 2313-2320, 2013.

[10] H. Wang and Y.-H. Tian, "Non-Lie symmetry groups and new exact solutions of a $(2+1)$-dimensional generalized Broer-Kaup system," Communications in Nonlinear Science and Numerical Simulation, vol. 16, no. 10, pp. 3933-3940, 2011.

[11] S. Kumar, K. Singh, and R. K. Gupta, "Painlevé analysis, Lie symmetries and exact solutions for $(2+1)$-dimensional variable coefficients Broer-Kaup equations," Communications in Nonlinear Science and Numerical Simulation, vol. 17, no. 4, pp. 1529-1541, 2012.

[12] A. Biswas, "Solitary wave solution for the generalized KdV equation with time-dependent damping and dispersion," Communications in Nonlinear Science and Numerical Simulation, vol. 14, no. 9-10, pp. 3503-3506, 2009.

[13] I. L. Freire and J. C. S. Sampaio, "Conservation laws for Kawahara equations," Matematica Aplicada E Computacional, 17 a 21 de setembro de 2012-Aguas de Lindola/SP.

[14] H. Liu, J. Li, and L. Liu, "Lie symmetry analysis, optimal systems and exact solutions to the fifth-order KdV types of equations," Journal of Mathematical Analysis and Applications, vol. 368, no. 2, pp. 551-558, 2010.

[15] L. Kaur and R. K. Gupta, "Kawahara equation and modified Kawahara equation with time dependent coefficients: symmetry analysis and generalized $G^{\prime} G$-expansion method," Mathematical Methods in the Applied Sciences, vol. 36, no. 5, pp. 584-600, 2013.

[16] O. Vaneeva, "Lie symmetries and exact solutions of variable coefficient $\mathrm{mKdV}$ equations: An equivalence based approach," Communications in Nonlinear Science and Numerical Simulation, vol. 17, no. 2, pp. 611-618, 2012.

[17] A.-M. Wazwaz, "Abundant solitons solutions for several forms of the fifth-order KdV equation by using the tanh method," Applied Mathematics and Computation, vol. 182, no. 1, pp. 283300, 2006.

[18] W. Chen, J. Li, C. Miao, and J. Wu, "Low regularity solutions of two fifth-order KDV type equations," Journal d'Analyse Mathematique, vol. 107, no. 1, pp. 221-238, 2009.

[19] H. Zhang, "Extended Jacobi elliptic function expansion method and its applications," Communications in Nonlinear Science and Numerical Simulation, vol. 12, no. 5, pp. 627-635, 2007.

[20] H. Liu and J. Li, "Lie symmetry analysis and exact solutions for the short pulse equation," Nonlinear Analysis: Theory, Methods and Applications, vol. 71, no. 5-6, pp. 2126-2133, 2009. 
[21] H. Liu, J. Li, and Q. Zhang, "Lie symmetry analysis and exact explicit solutions for general Burgers' equation," Journal of Computational and Applied Mathematics, vol. 228, no. 1, pp. 1-9, 2009.

[22] N. H. Asmar, Partial Differential Equations with Fourier Series and Boundary Value Problems, China Machine Press, Beijing, China, 2nd edition, 2005.

[23] H. Liu, J. Li, and L. Liu, "Group classifications, symmetry reductions and exact solutions to the nonlinear elastic rod equations," Advances in Applied Clifford Algebras, vol. 22, no. 1, pp. 107-122, 2012. 


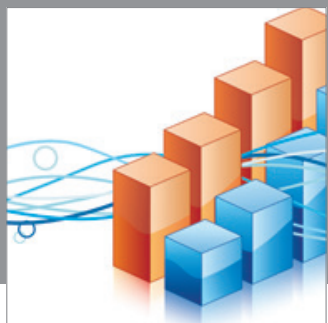

Advances in

Operations Research

mansans

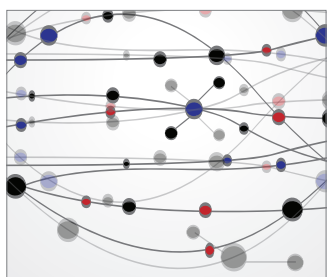

The Scientific World Journal
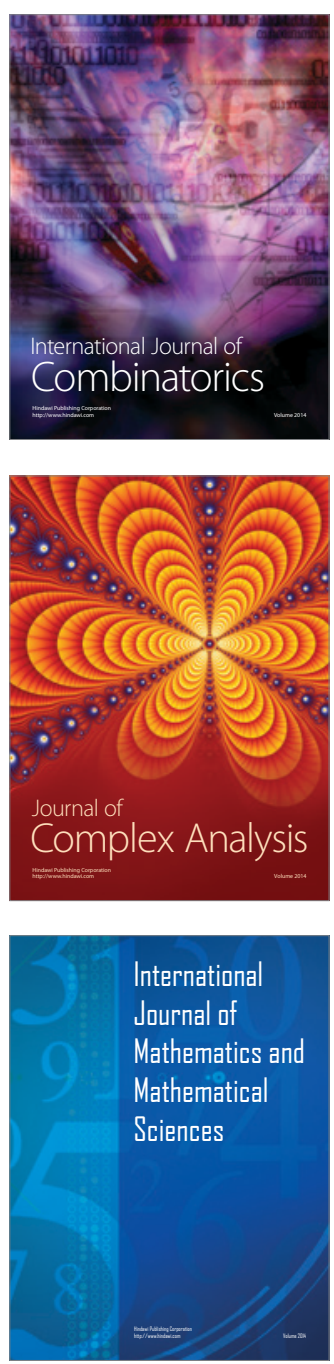
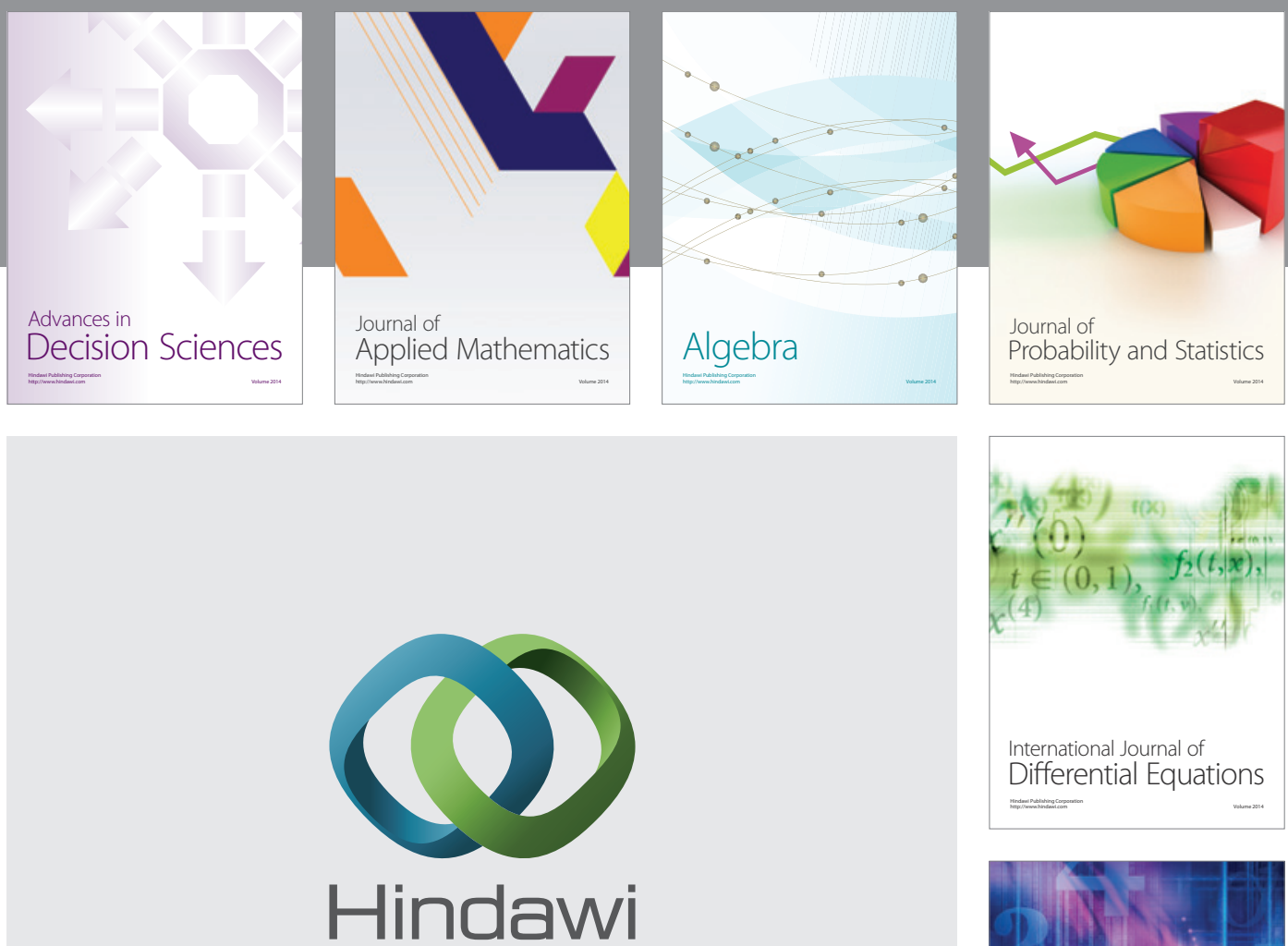

Submit your manuscripts at http://www.hindawi.com
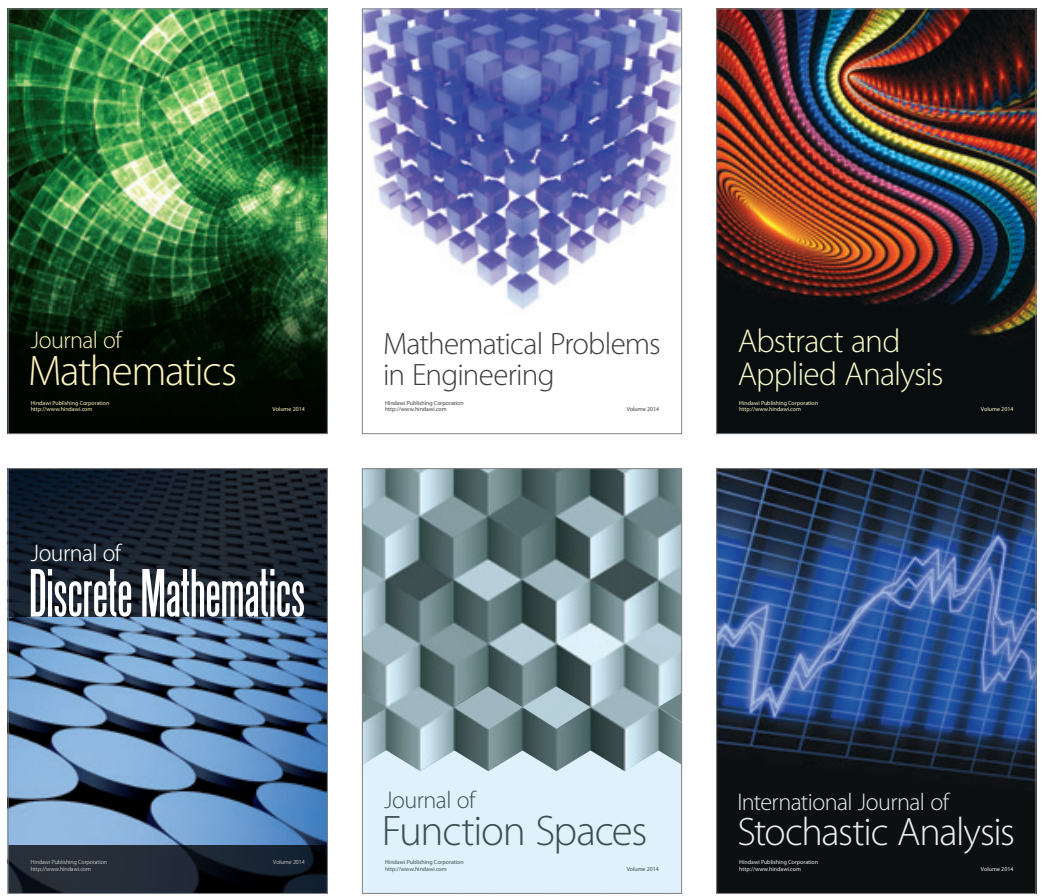

Journal of

Function Spaces

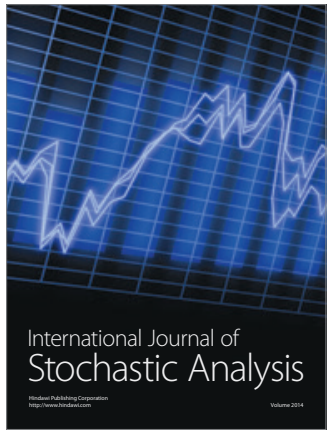

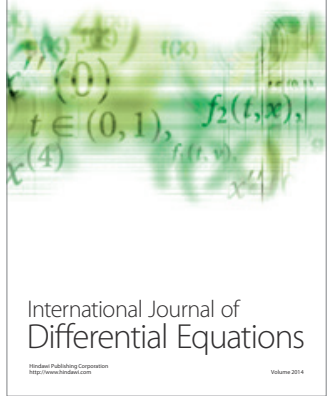
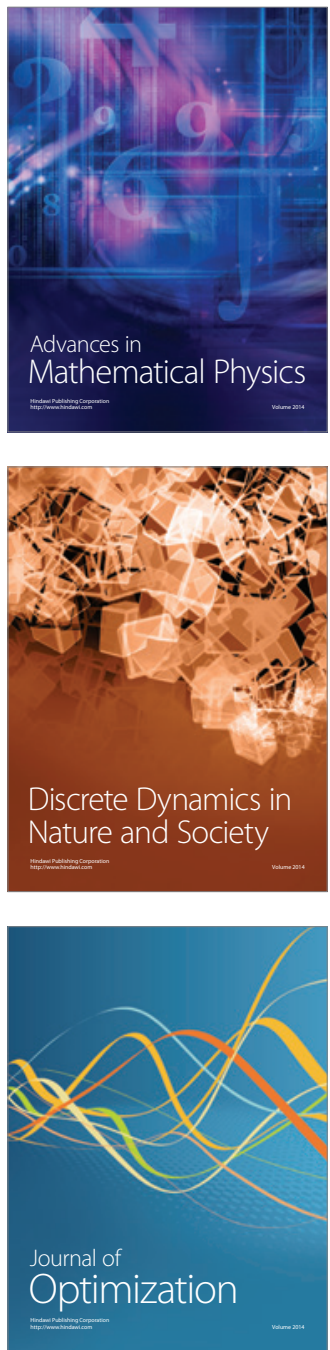